\title{
Super-Resolution for Computed Tomography Based on Discrete Tomography
}

\author{
Wim van Aarle, Kees Joost Batenburg, Gert Van Gompel, Elke Van de Casteele, and Jan Sijbers
}

\begin{abstract}
In computed tomography (CT), partial volume effects impede accurate segmentation of structures that are small with respect to the pixel size. In this paper, it is shown that for objects consisting of a small number of homogeneous materials, the reconstruction resolution can be substantially increased without altering the acquisition process. A super-resolution reconstruction approach is introduced that is based on discrete tomography, in which prior knowledge about the materials in the object is assumed. Discrete tomography has already been used to create reconstructions from a low number of projection angles, but in this paper, it is demonstrated that it can also be applied to increase the reconstruction resolution. Experiments on simulated and real $\mu \mathrm{CT}$ data of bone and foam structures show that the proposed method indeed leads to significantly improved structure segmentation and quantification compared with what can be achieved from conventional reconstructions.
\end{abstract}

Index Terms-Computed tomography, segmentation, superresolution, discrete tomography.

\section{INTRODUCTION}

I $\mathrm{N} X-R A Y$ Computed Tomography $(C T)$, images are typically reconstructed on a voxel grid. Since each voxel is represented by a constant grey level, it is intrinsically assumed that the material within such a voxel is homogeneous. It is clear, however, that a voxel representation cannot properly represent structures that have a varying density within a voxel. Thus, each voxel in the images could contain more than one material or tissue type. This phenomenon is referred to as the partial volume effect $(P V E)$. PVEs will cause object boundaries to be smeared out across the boundary voxels. Also, if a feature of the scanned object is small relative to the nominal voxel size, PVEs reduce the contrast between the structure of interest and

Manuscript received January 8, 2013; revised June 7, 2013, August 29, 2013, and November 26, 2013; accepted December 3, 2013. Date of publication January 2, 2014; date of current version February 4, 2014. This work was supported in part by iMinds, Interdisciplinary Institute for Technology, a Research Institute founded by the Flemish Government and in part by the IWT SBO Tomfood Project. The work of K. J. Batenburg was supported by the Netherlands Organisation for Scientific Research, Programme under Grant 639.072.005. The associate editor coordinating the review of this manuscript and approving it for publication was Prof. Jong Chul Ye.

W. van Aarle, E. Van de Casteele, and J. Sijbers are with the iMindsVisionlab, University of Antwerp, 2610 Antwerpen, Belgium (e-mail: wim.vanaarle@uantwerpen.be).

K. J. Batenburg is with the iMinds-Visionlab, University of Antwerp, Antwerpen 2610, Belgium, and also with Centrum Wiskunde and Informatica, 1098XG Amsterdam, The Netherlands (e-mail: joost.batenburg@uantwerpen.be).

G. Van Gompel is with the University Hospital of Brussels, Brussels 1083, Belgium (e-mail: gert.vangompel@uzbrussel.be).

Color versions of one or more of the figures in this paper are available online at http://ieeexplore.ieee.org.

Digital Object Identifier 10.1109/TIP.2013.2297025

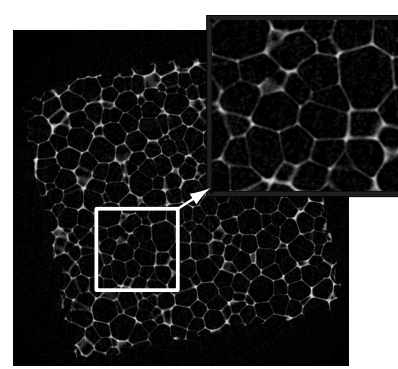

(a)

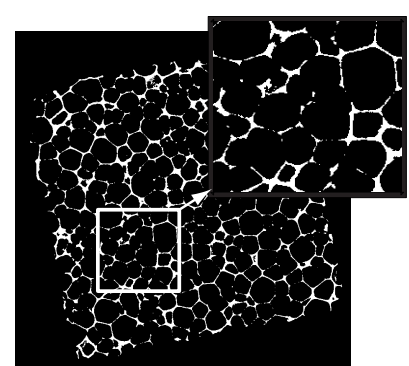

(b)
Fig. 1. (a) Reconstruction of a polyurethane foam, taken with a SkyScan $1172 \mu \mathrm{CT}$ scanner at a pixel resolution of $17 \mu \mathrm{m}$. (b) Otsu's segmentation of the reconstruction. Many cell walls remain undetected in the segmentation while other structures are overestimated.

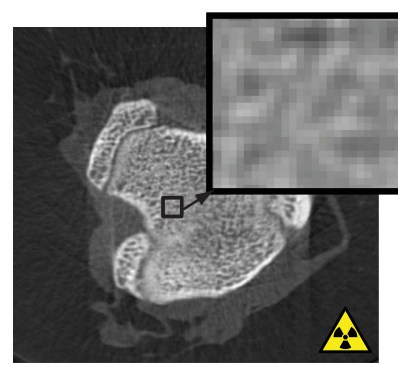

(a)

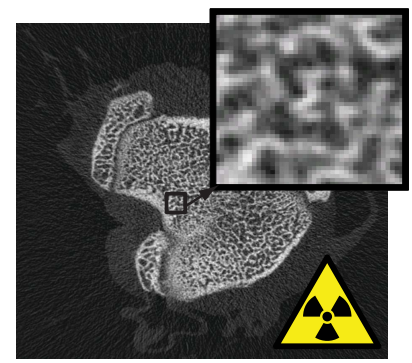

(b)
Fig. 2. FBP reconstructions of the epiphyseal plate of a rat femur taken at two different resolutions in a SkyScan $1172 \mu \mathrm{CT}$ scanner. (a) $35 \mu \mathrm{m}$ reconstruction, low radiation dose. (b) $9 \mu \mathrm{m}$ reconstruction, high radiation dose. This one is clearly much easier to segment. Note that as both slices were taken from different scans, the object was slightly displaced between the acquisition of both datasets. Even though image registration was performed, there is still a residual difference.

its background signal. Consequently, it is difficult to achieve the intrinsic resolution of the detector. Fig. 1(a) shows a filtered backprojection (FBP) reconstruction of a polyurethane foam for which the widths of the edges of the pores are comparable to the detector size. A globally thresholded segmentation of Fig. 1(a), created with the commonly used clustering method of Otsu [1], is shown in Fig. 1(b). Clearly, many thin structures remain undetected, whereas the thickness for some larger structures is overestimated.

To reduce PVEs, and hence to obtain sufficient contrast, a high resolution scan can be acquired. This, however, requires a much higher radiation dose and a longer scanning time [2]. In Fig. 2, an FBP reconstruction with a spatial resolution of $35 \mu \mathrm{m}$ is shown of a rat femur along with an FBP reconstruction with a spatial resolution of $9 \mu \mathrm{m}$ of the same femur. 
It is clear that the contrast in Fig. 2(b) is significantly better than that in Fig. 2(a). Fig. 2(b) is therefore better suited for accurate segmentation and estimation of the morphometric bone parameters [3], which is crucial to understand the effects of drug trials in for example osteoporosis research [4]. Other applications where high resolution images are required include metrology (e.g. to inspect the surface of the blades of aerofoil turbines), structural biology and materials research [5].

The conventional approach to reduce PVEs without increasing the radiation dose is to upsample the reconstruction voxel grid, allowing for a more accurate representation and potentially improving the overall visualisation of small structures. This upsampling is also known as super-resolution [6]. It is important to note, however, that in CT, a unique reconstructed image can only be obtained if the projection domain is adequately sampled. The required amount of information is dependent on the number of voxels. Upsampling the reconstruction grid therefore typically leads to a limited data reconstruction problem: the number of ray-equations (measured projection data) remains the same while the number of unknowns (reconstruction voxels) increases significantly. To overcome this problem, additional information must be entered into the reconstruction problem. This can be done in numerous ways. In [7]-[9], information from multiple low resolution CT images is combined into a high resolution CT image [7]-[9], but these methods result in an increased scan time and radiation dose. In [10], additional detector samples are created by Fourier interpolation and a compressed sensing solution is used to solve a reconstruction with many projection images and high noise intensity.

In this paper, a super-resolution approach for $\mathrm{CT}$ is proposed that effectively solves the limited data problem by incorporating prior knowledge about the unknown object. In CT, such prior knowledge comes in many forms, e.g. sparsity of the reconstructed image [11] or its gradient [12], [13]. Here, the novel super-resolution scheme is based on the Discrete Algebraic Reconstruction Technique (DART), an iterative reconstruction technique that can be applied if the scanned object is known to consist of a small set of materials, each corresponding to a different constant and priorly known grey level in the reconstruction [14]. Similar to [14], the focus in this work is on the reconstruction of images that consist of a small number of grey levels, typically up to 4 or 5 . It should be noted that in practice, due to the polychromatic nature of the X-ray spectrum, there is no quantitative model to exactly determine the grey levels based on the materials of the scanned object. However, it is typically possible to obtain a sufficiently accurate estimation by looking at a reconstructed image computed with a standard iterative technique such as SIRT [15]. Alternatively, in [16], [17], the authors provide methods to (semi-)automatically estimate grey level values for use in discrete tomography.

It has been shown already that by utilizing discrete tomography techniques, very accurate reconstructions can often be computed from only a few projection images [18]-[20]. In this paper, it will be demonstrated that discrete tomography can also be used for benefits in a different direction, namely to increase the resolution of the reconstructed images with the same (or only slightly less) number of projection angles. It will be shown that by upsampling the reconstruction grid and incorporating prior knowledge about the objects grey levels, the lack of high resolution projection data can be compensated. The proposed approach effectively increases the spatial resolution of the tomographic reconstructions [21], [22].

The paper is organized as follows. Section II introduces notation for algebraic and discrete tomography. Section III introduces the new super-resolution approach. Recent results [23] on provable error bounds for reconstructions are used to demonstrate that projections acquired at a certain resolution can in fact encode information of the original object at a higher resolution, making it necessary to use superresolution in the reconstruction. In Section IV, experiments are described that were performed to evaluate the reconstruction accuracy for the proposed super-resolution approach. Results are presented for both simulated data and experimental $\mu \mathrm{CT}$ data. Finally, Section V concludes this work.

\section{CONCEPTS AND NOTATION}

In this section, general concepts and notations are introduced. In Section II-A, the algebraic tomography model is described. For simplicity, a monochromatic x-ray beam will be assumed. Note, however, that this does not preclude application of the method to polychromatic $\mathrm{x}$-ray imaging since preprocessing methods can be applied to compute monochromatic from polychromatic projections [24]-[27]. Section II-B discusses algebraic reconstruction techniques such as SIRT. Section II-C concerns discrete reconstruction techniques such as DART, an iterative reconstruction technique that exploits prior knowledge about the grey levels of each of the scanned materials [14]. DART effectively combines reconstruction and segmentation into a single tomographic algorithm. For clarity, all concepts will be presented on a 2D parallel beam projection geometry. However, the proposed methods can be generalized to any acquisition geometry.

\section{A. Computed Tomography}

Let $f$ represent the 2D attenuation of a certain object, which will be referred to as the object function. A parallel beam projection geometry defines the tomographic projection of $f$ as the line integrals of $f$ along the lines $l_{\theta, t}=\{(x, y) \in$ $\mathbb{R} \times \mathbb{R}: x \cos \theta+y \sin \theta=t\}$, where $\theta \in[0, \pi)$ represents the angle between the line and the $y$-axis and where $t \in \mathbb{R}$ represents the coordinate along the projection axis. For a finite set of lines $l_{\theta, t}$, the X-ray beam intensity at the detectors, $I(\theta, t)$, are measured as

$$
I(\theta, t)=I_{0} e^{-\int_{l_{\theta, t}} f(x, y) d s(x, y)},
$$

with $I_{0}$ the incident beam intensity and $s(x, y)$ are all points along the line $l_{\theta, t}$. Define the attenuation projection function $p(\theta, t)$ as follows:

$$
p(\theta, t)=-\ln \left(\frac{I(\theta, t)}{I_{0}}\right)=\int_{l_{\theta, t}} f(x, y) d s(x, y),
$$

also called the forward projection or sinogram of $f(x, y)$. 
In practice, a projection is measured at a set of projection angles and at a set of detector elements with a width $\Delta t$. Let $\boldsymbol{I} \in \mathbb{R}^{m}$ denote the measured intensity data, with $m$ the number of detector values multiplied by the number of projection angles. For $j \in 1, \ldots, m, I_{j}$ can then be modelled as

$$
I_{j}=\int_{-\frac{\Delta t}{2}}^{\frac{\Delta t}{2}} I_{0} e^{-p\left(\theta, t+t^{\prime}\right)} d t^{\prime}
$$

with $t$ and $\theta$ the detector coordinate and projection angle of the measured detector value $I_{j}$, respectively. The attenuation projection data $\boldsymbol{p} \in \mathbb{R}^{m}$ can then be defined as follows:

$$
p_{j}=-\ln \left(\frac{I_{j}}{I_{0}}\right) \text {. }
$$

Note that, due to the logarithmic operation in Eq. (4), the contribution of a pixel to the measured projection values does not only depend on the average value of that pixel, but also on the distribution of the attenuation within that pixel.

Tomography deals with the reconstruction of $f(x, y)$ based on $\boldsymbol{p}$. This reconstructed function is represented by an image, a grid of square pixels with a finite width and height, $\Delta s$. Let $\boldsymbol{v} \in \mathbb{R}^{n}$ denote a discretized square image of the function $f(x, y)$, where $n$ denotes the number of pixels. $v_{i}$ can then be modelled as the total value of $f$, taken over the square pixel:

$$
v_{i}=\int_{-\frac{\Delta s}{2}}^{\frac{\Delta s}{2}} \int_{-\frac{\Delta s}{2}}^{\frac{\Delta s}{2}} f\left(x_{i}+x^{\prime}, y_{i}+y^{\prime}\right) d x^{\prime} d y^{\prime},
$$

with $x_{i}$ and $y_{i}$ the coordinates of the center point of pixel $v_{i}$.

The value of a certain pixel $v_{i}$ will thus depend on an entire area of values of the real object function. If the object has an edge running through the area of pixel $v_{i}$, or if the object is not homogeneous inside the pixel boundaries, the value of $v_{i}$ will not represent the attenuation coefficient of any of the materials of the object, but will represent an average of all attenuation coefficients. This is called the partial volume effect $(P V E)$. Note that for object functions that consist of piece-wise constant regions, the fraction of pixels for which PVEs occur is directly related to the size of $\Delta s$.

\section{B. Algebraic Tomography Model}

Using the discretized definitions of projection data (Eq. (4)) and reconstructed image (Eq. (5)), a computational model approximating the mathematical projection model — can be constructed. The forward projection of the object for a finite set of angles is modelled as a linear operator $\boldsymbol{W}$, called the projection operator, which maps the image $\boldsymbol{v}$ to the projection data $\boldsymbol{q}$ :

$$
\boldsymbol{q}:=\boldsymbol{W} \boldsymbol{v} .
$$

In Eq. (6), $\boldsymbol{W}=\left(w_{i j}\right)$ is an $m \times n$ matrix where $w_{i j}$ represents the contribution of image pixel $v_{j}$ to detector value $q_{i}$. The vector $\boldsymbol{q}$ is called the forward projection of $\boldsymbol{v}$. The reconstruction problem in CT can then be modelled as the recovery of $\boldsymbol{v}$ from a given vector $\boldsymbol{p}$ of projection data, such that:

$$
W v=p .
$$

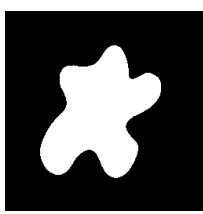

(a)

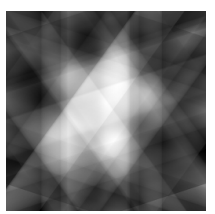

(b)

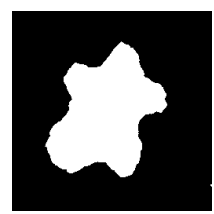

(c)

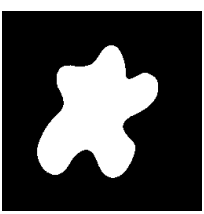

(d)
Fig. 3. (a) $256 \times 256$ phantom image. (b) SIRT reconstruction using 5 equiangular projections. (c) Segmentation of (b) using Otsu's method [1] (S-SIRT). (d) DART reconstruction using 5 equiangular projections.

Many reconstruction algorithms have been proposed to solve Eq. (7) without any constraints on $v$ [28]. One of these methods is the Simultaneous Iterative Reconstruction Technique (SIRT), which will be used, as defined in [15], throughout this paper. SIRT is a linear algorithm that finds a solution $\tilde{v}$ such that the weighted squared projection difference $\| \boldsymbol{W} \tilde{\boldsymbol{v}}-$ $\boldsymbol{p} \|_{R}=(\boldsymbol{W} \tilde{\boldsymbol{v}}-\boldsymbol{p})^{T} \boldsymbol{R}(\boldsymbol{W} \tilde{\boldsymbol{v}}-\boldsymbol{p})$ is minimal. $\boldsymbol{R} \in \mathbb{R}^{m \times m}$ is a diagonal matrix that contains the inverse row sums of $\boldsymbol{W}$ : $r_{i i}=1 / \sum_{j} w_{i j}$. In each iteration $k$, the current reconstruction $\boldsymbol{v}^{(k-1)}$ is updated, yielding a new reconstruction $\boldsymbol{v}^{(k)}$, as follows:

$$
v_{j}^{(k)}=v_{j}^{(k-1)}+\lambda \frac{1}{\sum_{i=1}^{m} w_{i j}} \sum_{i=1}^{m} \frac{w_{i j}\left(p_{i}-w_{i j} v_{i}^{(k-1)}\right)}{\sum_{h=1}^{n} w_{i h}} .
$$

In Eq. (8), $\lambda$ is a relaxation parameter.

\section{Discrete Tomography}

Discrete tomography deals with the reconstruction of objects with a limited number of different materials, and uses this information to restrict the number of possible greylevels in the reconstructed images. Many methods for discrete tomography exist, most of which are built on the theoretical frameworks of Herman and Kuba [29], [30]. In this work, the Discrete Algebraic Reconstruction Technique (DART) algorithm is chosen as it has shown great potential in practical applications [5], [31], [32].

In some reconstruction problems $m$ is much smaller than $n$ (e.g. when the number of projection directions is very low or the data is truncated), which leads to an underdetermined system of linear equations: so-called limited data problems. Fig. 3(b) shows a SIRT reconstructed image of the phantom image in Fig. 3(a) from only 5 equiangular projections. The segmentation of Fig. 3(b) using Otsu's method [1], which will be referred to by S-SIRT in the remainder of this paper, is shown in Fig. 3(c). Note that mainly the pixels near the border or the object are incorrectly segmented.

The observation is used by DART to reduce the size of Eq. (7). DART uses prior knowledge about the discrete grey levels to iteratively solve Eq. (7) under the constraint that $v_{i}$ can only take values that are elements of a set $\rho=\mathbb{R}^{l}$. Each element of $\rho$ contains the grey level value of one of the $l$ different materials of the scanned object. It is chosen by the user based on the available prior knowledge.

Here, a concise summary of the algorithmic steps in DART is given. For any $A \subset\{1, \ldots, n\}$, and any $\overline{\boldsymbol{v}} \in \mathbb{R}^{n}$, let $\overline{\boldsymbol{v}}_{A} \in \mathbb{R}^{n_{A}}$ be a vector that contains a subset of the entries of $\overline{\boldsymbol{v}}$, where 


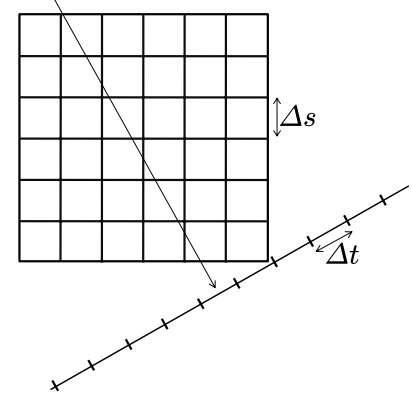

(a)

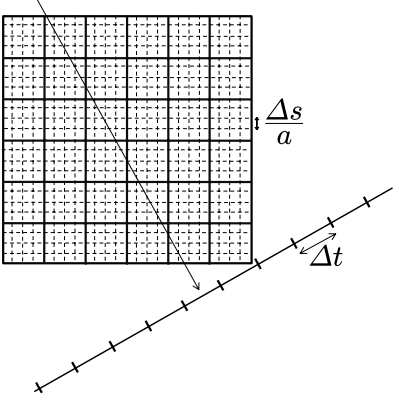

(b)

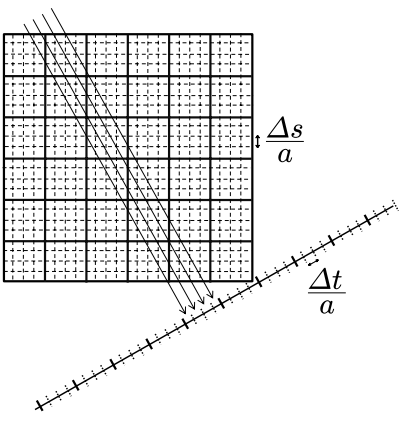

(c)

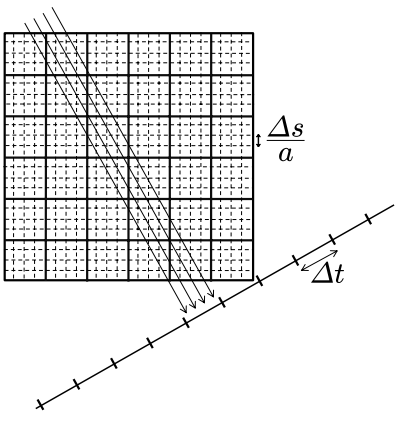

(d)

Fig. 4. (a) Basic projection geometry. Each detector cell corresponds to a single ray. (b) Upsampled reconstruction grid. Certain pixels are not hit by a ray. (c) Sinogram upsampling. Each detector is subdivided into multiple detectors with interpolated values (d) Detector supersampling. Each detector corresponds to multiple rays. The contribution of each ray is summed.

$\bar{v}_{i}$ is included iff $i \in A$. Furthermore, let $\boldsymbol{W}_{A} \in \mathbb{R}^{m \times n_{A}}$ be the matrix that contains the columns $i \in A$ of the matrix $\boldsymbol{W}$. The DART algorithm consists of the following steps:

1) Create an initial reconstruction $\boldsymbol{v}^{(0)}$ using SIRT. Put $k=0$, the iteration number.

2) If $k>0$, apply a smoothing filter to $\boldsymbol{v}^{(k)}$. This can be done by application of a convolution with the 2D stencil $\frac{b}{8}[111 ; 1(1-b) 1 ; 111]$, where $b$ is the intensity of the smoothing, which is typically chosen at $b=0.20$. This smoothing step is required because if pixels can very independently of each other, great variations near the border tend to occur. Blurring then regularizes the data.

3) Segment $\boldsymbol{v}^{(k)}$. A simple scheme with fixed global thresholds, $\boldsymbol{\tau} \in \mathbb{R}^{l-1}$, is used to replace the grey level of each pixel $v_{i}^{(k)}$ by that of the corresponding value of $\rho$. The values $\tau$ are typically chosen exactly in the middle of two grey levels.

4) Determine $A \subset\{1, \ldots, n\}$, the set of border pixels. A pixel is defined as a border pixel if its value is different from any of its neighbours (defined by an 8-connectivity window). To allow non-border pixels to be updated as well and to further reduce the impact of noisy projection data, a small number of additional random pixels are added to A (typically about $10 \%$ ).

5) Compute $\boldsymbol{p}^{(k+1)}$, the residual projection data, by subtracting the forward projection of all pixels $v_{i}^{(k)}$ with $i \notin A$ from the measured data $\boldsymbol{p}$.

6) Create the reconstruction $\boldsymbol{v}^{(k+1)}$ using SIRT by solving $\boldsymbol{W}_{A} \boldsymbol{v}_{A}^{(k+1)}=\boldsymbol{p}^{(k+1)}$. This system of equations has a much smaller number of unknowns than the original system and is therefore better determined, even when few projection angles are available.

7) Increase $k$ by 1 and return to step 2 until some termination criterion has been reached.

For a more in depth description of DART, we refer to the original publication [14]. Fig. 3(d) shows a DART reconstruction of the phantom image in Fig. 3(a) from only 5 projections.

Note that, from its design, DART is especially suited for structures that are large with respect to $\Delta s$. If the object to be reconstructed consists of many small structures, such as foams

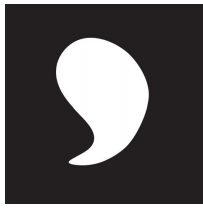

(a)

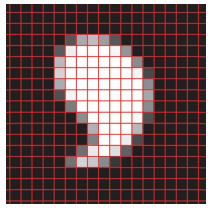

(b)

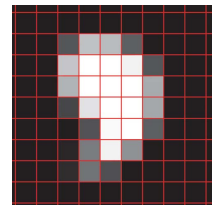

(c)

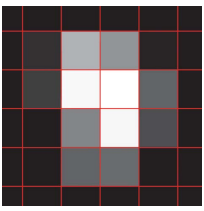

(d)
Fig. 5. (a) A binary object. (b)-(d) The same binary object, represented on a pixel grid with increasing pixel sizes.

or trabecular bone, two effects limit the possible improvements of DART over standard techniques. For one, the PVE breaks the assumption that the number of grey levels is small. Even binary objects can then no longer be accurately depicted on a grid with only black or white pixel values (Fig. 5). Also, for small objects, the number of elements in $A$ will still be large, thereby insufficiently reducing the reconstruction problem size.

\section{SUPER-RESOLUTION}

To achieve the intrinsic detector resolution and to counter the PVE, the reconstruction grid must be upsampled [Fig. 4(b)]. Let $a$ be the upsampling factor in each dimension. Each pixel of width $\Delta s$ is then subdivided into $a^{2}$ pixels of width $\frac{\Delta s}{a}$. Denote the upsampled reconstruction image by $\boldsymbol{v}^{\prime} \in \mathbb{R}^{a^{2} n}$.

Note that, typically, $\frac{\Delta s}{a}$ is different from $\Delta t$, the width of the detector cell. If the projection weights $w_{i j}$ are computed by intersection of a single ray with the upsampled image, some pixels will not have a ray going through them for each projection angle and the projection data will not be computed correctly. Two methods are investigated to overcome this problem: sinogram upsampling [Fig. 4(c), Section III-A] and detector supersampling [Fig. 4(d), Section III-B].

Define the relative reconstruction resolution as the ratio of the detector width, $\Delta t$, to the pixel size, $\Delta s$ :

$$
R_{v}=\frac{\Delta t}{\Delta s} \text {. }
$$

\section{A. Sinogram Upsampling}

With sinogram upsampling ( $S U$ ), the number of detector cells is artificially increased by subdividing each detector of 

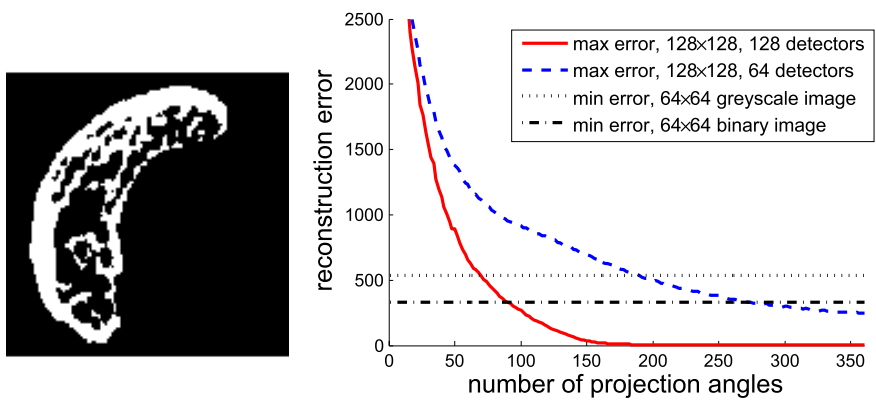

(a)

(b)

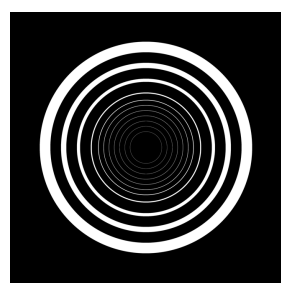

(a)

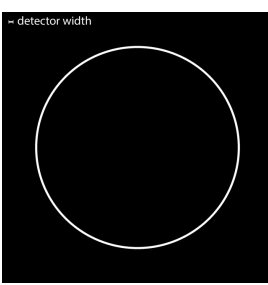

(b)

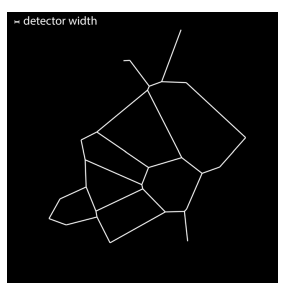

(c)

Fig. 7. Rasterized versions of the analytical binary phantoms used in the experimental section. (a) Rings phantom 1. (b) Ring phantom 2 e.g. $R_{p}=$ $\frac{1}{2}$. (c) Foam phantom e.g. $R_{p}=\frac{1}{2}$.

Fig. 6. (a) A $128 \times 128$ binary object. (b) In red and blue, the maximal error of any solution to $\boldsymbol{W} \boldsymbol{x}=\boldsymbol{p}$, as a function of the number of projection angles. In black, the minimal error for any image represented on the coarse $64 \times 64$ grid.

size $\Delta t$ into $a$ detectors of size $\frac{\Delta t}{a}$. Fig. 4(c) shows a schematic overview of this geometry. The value of each detector point is determined by linear interpolation of $\boldsymbol{p}$. Let $\boldsymbol{p}^{\prime} \in \mathbb{R}^{a m}$ be the upsampled sinogram and let $\boldsymbol{W}_{S U} \in \mathbb{R}^{a m \times a^{2} n}$ be the corresponding projection operator. The reconstruction equation then becomes:

$$
\boldsymbol{W}_{S U} \boldsymbol{v}^{\prime}=\boldsymbol{p}^{\prime}
$$

Note that, the relative reconstruction resolution, $R_{v^{\prime}}$, has remained the same. Furthermore, when interpolating the projection data, a certain smoothness in the projection data is assumed.

\section{B. Detector Supersampling}

With detector supersampling $(D S)$, the sinogram $\boldsymbol{p}$ remains unaltered, and no interpolation is thus required. However, the number of virtual rays targeting each detector cell is increased by a factor $a$, each $\frac{\Delta t}{a}$ apart. The relative reconstruction resolution increases by the same factor. Fig. 4(d) shows a schematic overview of this geometry. The reconstruction equation is:

$$
\boldsymbol{W}_{D S} \boldsymbol{v}^{\prime}=\boldsymbol{p},
$$

where each row in the projection operator $\boldsymbol{W}_{D S} \in \mathbb{R}^{m \times a^{2} n}$ is the summation of the $a$ corresponding rows of $\boldsymbol{W}_{S U} \boldsymbol{v}^{\prime}$.

In Eq. (10) and Eq. (11) the number of unknowns has been increased by a factor $a^{2}$ while the number of equations has been increased by a factor $a$ (Eq. (10)) and remained unaltered (Eq. (11)), respectively. Solving the reconstruction equation is now a limited data problem. As was noted in Section II, prior knowledge about the scanned objects can be used to solve Eq. (10) and Eq. (11) with the DART algorithm.

Note that there is a non-linear relationship between the measured projection data $\boldsymbol{p}$ and the actual attenuation projection data $p(\theta, t)$ [Eq. (3) and Eq. (4)]. As DART uses a linear projection model, the proposed super-resolution approaches do not accurately model the PVE. In the next section, however, it will be experimentally demonstrated that even with this limited model, super-resolution on piecewise homogeneous objects with known attenuation coefficients can indeed be achieved, leading to significant improvements in reconstruction accuracy.

\section{Error Bounds for Super-Resolution Reconstructions}

In a recent publication [23], a method is described for computing provable upper bounds on the maximum error of solutions to binary tomography problems with respect to the unknown original binary object. Here, this work will be used to prove for a parallel beam geometry that increasing the resolution of the reconstruction beyond the intrinsic detector resolution allows for more accurate reconstructions than the best possible reconstruction at the standard resolution. In other words: the projection data at the detector resolution contains sufficient information to accurately approximate the original object on a finer resolution grid.

Let $\tilde{v} \in\{0,1\}^{n}$ be a binary image. Hence

$$
\|\tilde{\boldsymbol{v}}\|_{1}=\|\tilde{\boldsymbol{v}}\|_{2}^{2} \text {. }
$$

Assume that $\boldsymbol{p}$ represents the perfect, non-truncated and noiseless projections of the original image $\tilde{\boldsymbol{v}}$, i.e. $\boldsymbol{p}:=\boldsymbol{W} \tilde{\boldsymbol{v}}$. For a parallel beam geometry the summed intensity measured at the detector for any angle equals the summed intensity of the original object. Hence

$$
\|\boldsymbol{p}\|_{1}=k\|\tilde{\boldsymbol{v}}\|_{1},
$$

where $k$ is the number of projection angles. For any solution $\bar{v}$ to Eq. (7), it then follows that

$$
\|\overline{\boldsymbol{v}}\|_{2}^{2}=\frac{\|\boldsymbol{p}\|_{1}}{k} .
$$

Let $\boldsymbol{v}^{*}=\boldsymbol{W}^{+} \boldsymbol{p}$ denote the least squares solution of Eq. (7), where $\boldsymbol{W}^{+}$is the Moore-Penrose pseudoinverse. The vector $\boldsymbol{v}^{*}$ can be computed by explicitly forming $\boldsymbol{W}^{+}$, or iteratively by using the Conjugate Gradient Least Squares (CGLS) technique. In [23], it is proved that for each $\overline{\boldsymbol{v}}_{\mathbf{1}}, \overline{\boldsymbol{v}}_{\mathbf{2}} \in\{0,1\}^{n}$ with $W \bar{v}_{1}=W \bar{v}_{2}=p$ :

$$
d\left(\overline{\boldsymbol{v}}_{\mathbf{1}}, \overline{\boldsymbol{v}}_{\mathbf{2}}\right) \leq 4\left(\left\|\overline{\boldsymbol{v}}_{\mathbf{1}}\right\|_{2}^{2}-\left\|\boldsymbol{v}^{*}\right\|_{2}^{2}\right),
$$

where $d\left(\overline{\boldsymbol{v}}_{\mathbf{1}}, \overline{\boldsymbol{v}}_{\mathbf{2}}\right)$ is the Euclidean distance between $\overline{\boldsymbol{v}}_{\mathbf{1}}$ and $\overline{\boldsymbol{v}}_{\mathbf{2}}$. Given that $\tilde{\boldsymbol{v}}$ and $\overline{\boldsymbol{v}}$ are binary images, $d(\tilde{\boldsymbol{v}}, \overline{\boldsymbol{v}})=\|\tilde{\boldsymbol{v}}-\overline{\boldsymbol{v}}\|_{2}^{2}$, and

$$
\max _{\overline{\boldsymbol{v}} \text { with } \boldsymbol{W} \overline{\boldsymbol{v}}=\boldsymbol{p}}\|\tilde{\boldsymbol{v}}-\overline{\boldsymbol{v}}\|_{2}^{2} \leq 4\left(\frac{\|\boldsymbol{p}\|_{1}}{k}-\left\|\boldsymbol{v}^{*}\right\|_{2}^{2}\right),
$$

an upper bound that can be easily computed.

For binary objects that can be fully represented on the reconstruction grid, the minimal error of any solution is of course zero. 


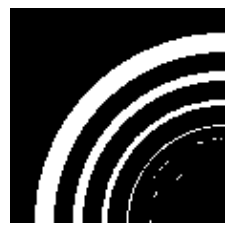

(a) DS, $a=1$ DART

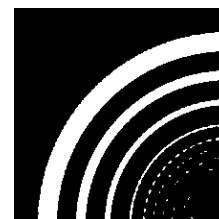

(b) DS, $a=2$ DART

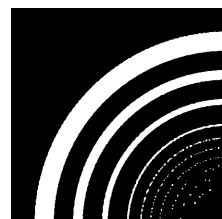

(c) DS, $a=4$ DART

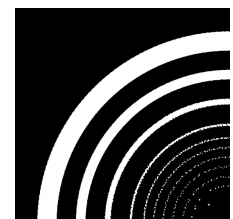

(d) DS, $a=10$ DART

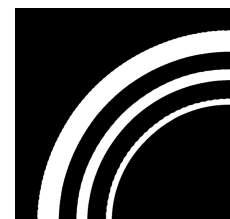

(e) SU, $a=10$ S-SIRT

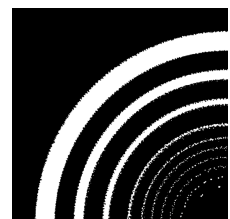

(f) $\mathrm{SU}, a=10$ DART

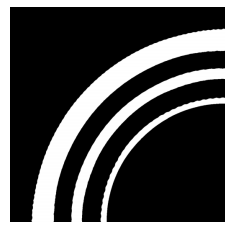

(g) DS, $a=10$ S-SIRT

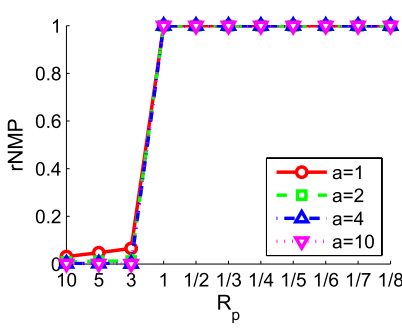

(h) SU, S-SIRT

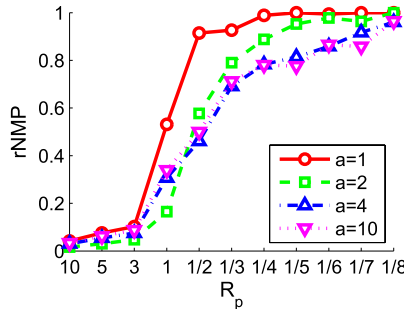

(i) SU, DART

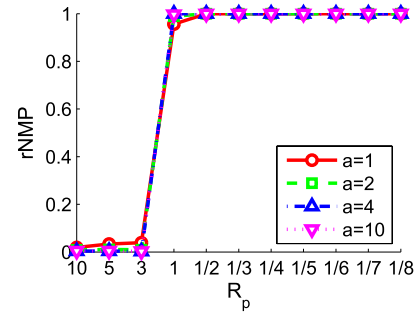

(j) DS, S-SIRT

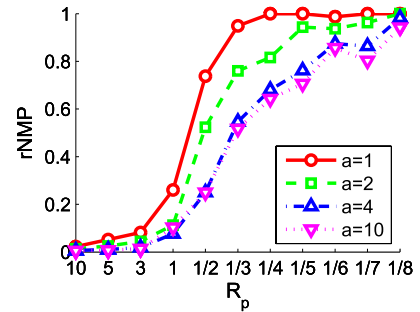

(k) DS, DART

Fig. 8. Experimental results from a simulated analytical phantom containing 11 rings of varying width. (a) High resolution rendering of the phantom image, also used as ground truth image. (b)-(d) The inner rings become more visible as $a$ increases. (e)-(h) Reconstructions of each proposed super-resolution approach, with (DART) and without (S-SIRT) prior knowledge. (i)-(1) For increasing values of $a$, plotting the relative Number of Misclassified Pixels (rNMP) in function of the widths of each ring.

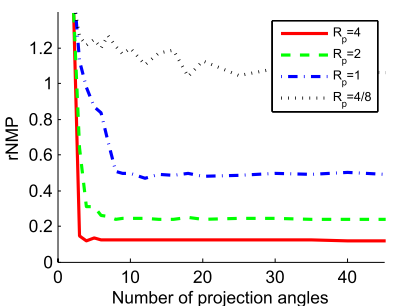

(a) Fig. $7 \mathrm{~b}, a=1$

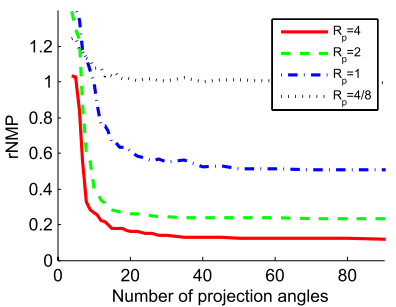

(e) Fig. $7 \mathrm{c}, a=1$

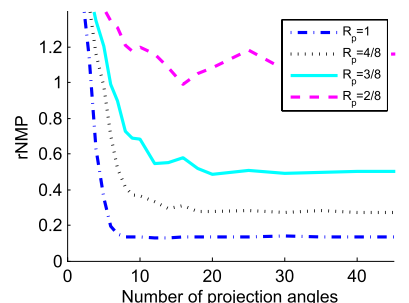

(b) Fig. 7b, $a=2$

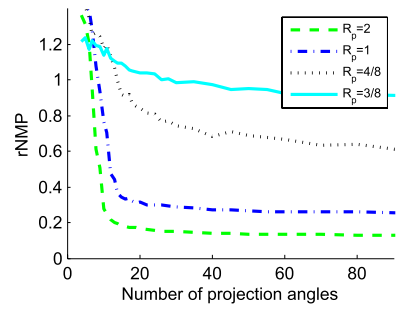

(f) Fig. 7c, $a=4$

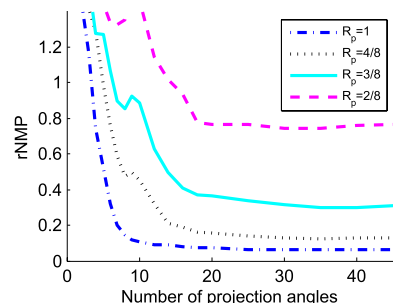

(c) Fig. $7 \mathrm{~b}, a=8$

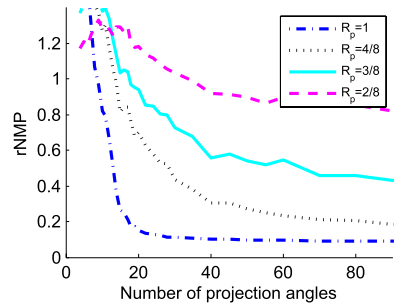

(g) Fig. 7c, $a=16$

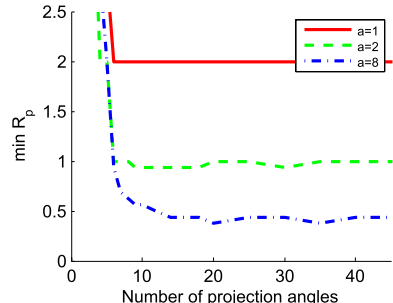

(d) Fig. $7 \mathrm{~b}, \min R_{p}$

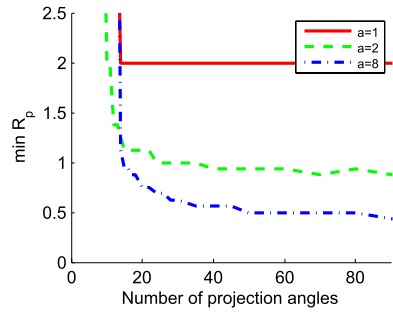

(h) Fig. 7c, $\min R_{p}$

Fig. 9. Experimental results from simulated analytical structures (a-d: Fig. 7; e-h: Fig. 7) with a varying thickness $\Delta q$. (a)-(c), (e)-(g) For different levels up upsampling, the rNMP of the objects with varying thickness as a function of the number of projection angles. After a certain point, additional projections offer no further accuracy improvements. This point is dependent on the thickness of the rings and on the level of upsampling. (d) and (h) The minimal $R_{p}$ of the structures that can be reconstructed with rNMP $<0.30$ as a function of the projection count. Higher levels of upsampling clearly result in better resolution but to reach the point of "sufficient information", more projections are required.

If the object $\bar{v}$ has to be represented on a grid that is too coarse (e.g. on $\frac{n}{a^{2}}$ instead of $n$ pixels with $a \in \mathbb{N}, a>1$ ), it will likely contain various grey levels. The initial assumption then no longer holds and a maximal error bound can no longer be computed. In this case, however, if the original object is known, the minimal error of any reconstruction can still be computed. Let $\tilde{\boldsymbol{v}}^{\prime} \in \mathbb{R}^{\frac{n}{a^{2}}}$ be a representation of $\tilde{\boldsymbol{v}}$ on a coarser grid where each pixel takes the average of the pixels on the finer grid, and let $\tilde{\boldsymbol{v}}^{\prime \prime} \in \mathbb{R}^{n}$ be its re-upsampling (with nearest neighbour interpolation) to the original grid. The minimal error for any vector $\overline{\boldsymbol{v}}^{\prime \prime}$ that is an $a$-times upsampled version of a vector on the coarse grid, is then

$$
\min _{\overline{\boldsymbol{v}}^{\prime \prime}}\left\|\tilde{\boldsymbol{v}}-\overline{\boldsymbol{v}}^{\prime \prime}\right\|_{2}^{2}=\left\|\tilde{\boldsymbol{v}}-\tilde{\boldsymbol{v}}^{\prime \prime}\right\|_{2}^{2}
$$

Note that this minimal error is closely related to the PVE that is introduced by representing $\tilde{\boldsymbol{v}}$ on the coarse grid. If the image $\tilde{\boldsymbol{v}}^{\prime}$ is segmented by thresholding before re-upsampling, the minimal error of any image that is an $a$-times upsampled version of a binary vector on the coarse grid can be found analogously.

In Fig. 6(b), the discussed error bounds are plotted as a function of $k$, the number of projection angles, for the $128 \times 128$ 

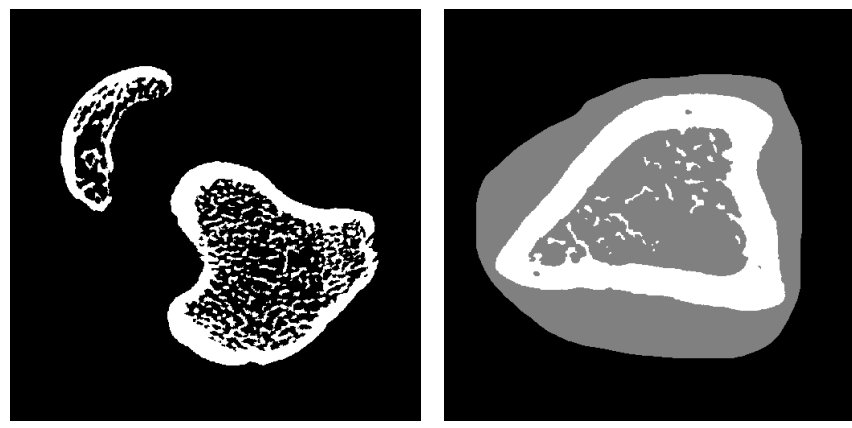

(a) bone 1 (binary)

(b) bone 2 (3 grey levels)

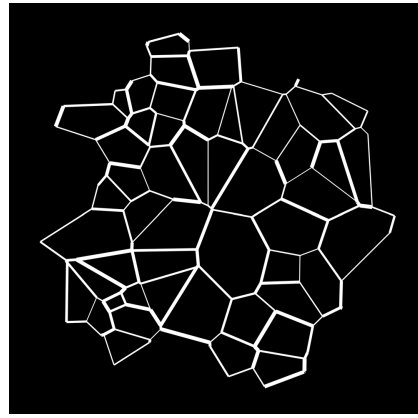

(c) one of 20 foams (binary)

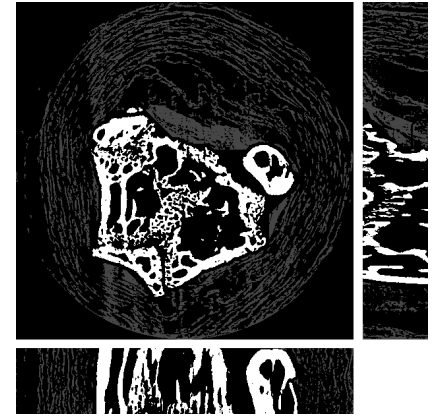

(d) bone 3 (3 grey levels, 3D)

Fig. 10. Simulated phantom images. Projection data of phantom (a), (b) and (d) was generated from high resolution pixel images, based on actual reconstructions of rat femurs. The set of phantoms (c) were analytically defined and their projection data were also calculated analytically. (a) Bone 1 (binary). (b) Bone 2 (3 grey levels). (c) One of 20 foams (binary). (d) Bone 3 (3 grey levels, 3D).

binary phantom object depicted in Fig. 6(a). In red, it shows that the maximal error of any solution that is computed from projection data with 128 detector points per direction, clearly drops as more projections become available. The same can be said of the blue line, which plots the maximal error of any solution computed from projection data with only 64 detectors, which required the use of the previously discussed detector supersampling technique (without DART). In the two black lines, the minimal error [Eq. (17)] is plotted for any greyscale and binary $64 \times 64$ image.

The analysis shows that if enough data is available, the maximal error of any solution computed on a sufficiently fine grid, can be smaller than the minimal error that can be expected for reconstructions on a grid that is too coarse for the original object. This suggests that in practical cases, where objects can typically only be correctly represented on grids with infinitesimally small pixels, it is indeed highly useful to compute reconstructions on grids that are much finer than the intrinsic detector resolution. This can be done using a super-resolution approach, such as the ones discussed in Section III-A and Section III-B, combined with a limited data solving reconstruction method, such as DART.

\section{EXPERIMENTS}

In this section, the proposed super-resolution method is demonstrated and its effectiveness is evaluated on various simulated images (Section IV-A) and on real datasets (Section IV-B).

\section{A. Simulation Experiments}

Experiments were performed on several simulation phantoms (Figs. 7 and 10). Projection data was generated analytically for some, and based on high resolution rasterized images for others. The relative Number of Misclassified Pixels (rNMP) is used as a performance metric. The rNMP measures the total number of pixels that are classified in a wrong partition (false negatives as well as false positives) with respect to the total number of pixels of that object. For analytical phantom images, the rNMP values were approximated by comparing the reconstructions to a very high resolution rasterization of the phantom images. Additionally, all experiments in this paper have also been evaluated with the Root Mean Square Error (RMSE) and the Structural Similarity (SSIM) validation metrics, which is defined in [33]. These additional metrics score different aspects of the accuracy. Their results, however, provided similar insights into the performance of the methods as the rNMP. For the sake of brevity, only rNMP is therefore covered here.

Analytical phantom 1: In the first experiment, the efficacy of a discrete super-resolution technique was examined as a function of the size of a structure with respect to $\Delta t$. To this end, a simulated analytical binary phantom containing 11 rings with a varying width, $\Delta q$, was created. A rasterized rendering of this phantom is depicted in Fig. 7(a). As a measure of the magnitude of the PVE, the notion of relative projection resolution, $R_{p}$, is introduced. It is defined as the ratio of the object width, i.e the thickness of the ring, to the detector width:

$$
R_{p}=\frac{\Delta q}{\Delta t}
$$

For the phantom in Fig. 7(a), the $R_{p}$ of the outer three rings is 10,5 and 3 . The $R_{p}$ of the fourth ring is 1 and can thus be used to measure if the intrinsic detector resolution is achieved. The seven most inner rings have an $R_{p}$ of $\frac{1}{2}$ to $\frac{1}{8}$.

Projection data was analytically generated [using Eq. (4)] for a parallel beam geometry with 60 equiangular projection angles and 256 detector pixels. Reconstructions were computed for both S-SIRT and DART and with both the sinogram upsampling approach and the detector supersampling approach, with increasing levels of super-resolution: $a=1,2$, 4 and 10.

From Fig. 8(a)-(d), it is clear that by increasing $a$ (combined with DS and DART), the spatial resolution improves, as indicated by the appearance of the rings in the center. This effect is less pronounced if $\mathrm{SU}$ is used [Fig. 8(f)]. If no prior knowledge is included in the reconstruction [Fig. 8(e) and (g)], the thin rings can not be seen at all. These results can also be observed in Fig. 8h-k, where for each $a$ the rNMP of each ring is plotted. For this experiment, the rNMP was computed for each ring separately. The false negatives of each ring can be easily counted, but counting false positive pixels is more difficult as it is not clear to which ring such pixel belongs. In the results shown in Fig. 8, each false positive pixel is accounted to the ring that it is closest to.

Analytical phantoms 2: In a second experiment, it is explored how the number of required projection angles is related to the level of volume upsampling, used in combination 


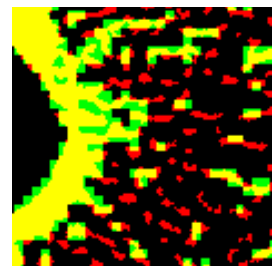

(a) $a=1$

S-SIRT

$\mathrm{rNMP}=0.388$

SSIM $=0.8189$

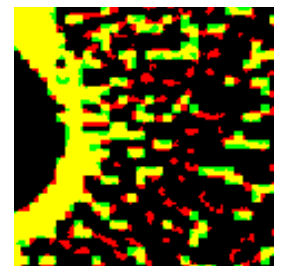

(b) $a=1$

DART

rNMP $=0.460$

SSIM $=0.8197$

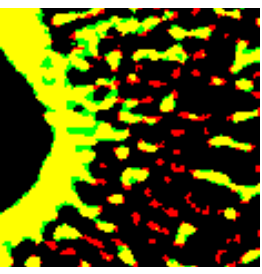

(c) SU, $a=4$

S-SIRT

$\mathrm{rNMP}=0.249$

SSIM $=0.8697$

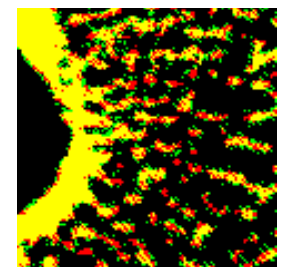

(d) SU, $a=4$

DART

$\mathrm{rNMP}=0.302$

$\mathrm{SSIM}=0.8483$

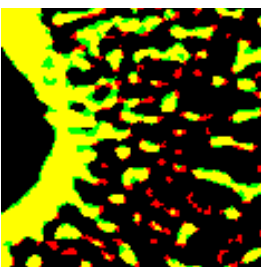

(e) DS, $a=4$

S-SIRT

$\mathrm{rNMP}=0.208$

SSIM $=0.8862$

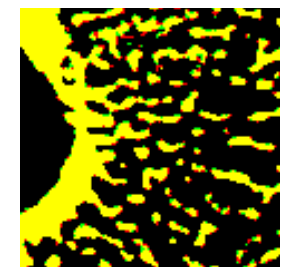

(f) DS, $a=4$

DART

rNMP $=0.086$

SSIM=0.9545

Fig. 11. Region of the various reconstructions of Fig. 10(a) on a parallel beam geometry with 180 projection direction and $I_{0}=20000$. The ground truth image is displayed in red and the reconstructions in green. Where both images overlap, i.e. where the segmentation is correct, the corresponding pixel is yellow.

\begin{tabular}{|c|c|c|c|c|c|c|c|c|c|c|c|c|c|c|}
\hline \multirow{3}{*}{ Phantom } & \multicolumn{7}{|c|}{ rNMP } & \multicolumn{7}{|c|}{ SSIM } \\
\hline & \multicolumn{2}{|c|}{$a=1$} & \multicolumn{3}{|c|}{ SU } & \multicolumn{2}{|c|}{ DS } & \multicolumn{2}{|c|}{$a=1$} & \multicolumn{3}{|c|}{ SU } & \multicolumn{2}{|c|}{ DS } \\
\hline & S-SIRT & DART & $a$ & S-SIRT & DART & S-SIRT & DART & S-SIRT & DART & $a$ & S-SIRT & DART & S-SIRT & DART \\
\hline Fig. 10a & 0.388 & 0.460 & 4 & 0.249 & 0.302 & 0.208 & 0.086 & 0.8189 & 0.8197 & 4 & 0.8697 & 0.8483 & 0.8862 & 0.9545 \\
\hline Fig. 10b & 0.519 & 0.036 & 4 & 0.519 & 0.026 & 0.519 & 0.003 & 0.8108 & 0.8952 & 4 & 0.8198 & 0.9106 & 0.8275 & 0.9859 \\
\hline Fig. 10c & 0.468 & 0.347 & 8 & 0.463 & 0.381 & 0.389 & 0.107 & 0.9971 & 0.9981 & 8 & 0.9972 & 0.9984 & 0.9978 & 0.9997 \\
\hline Fig. 10d & 0.168 & 0.172 & 4 & 0.112 & 0.090 & 0.091 & 0.068 & 0.8980 & 0.8987 & 4 & 0.9012 & 0.9134 & 0.9115 & 0.9834 \\
\hline
\end{tabular}

Fig. 12. Numerical results (rNMP and SSIM) for all phantom experiments of Fig. 10. For the set of phantom Fig. 10(c), the average values are given. A parallel beam geometry with 180 projection direction and $I_{0}=20000$ was used.

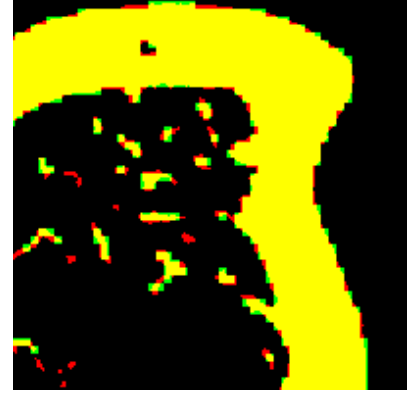

(a) $a=1$, DART

$\mathrm{rNMP}=0.036$

SSIM $=0.8952$

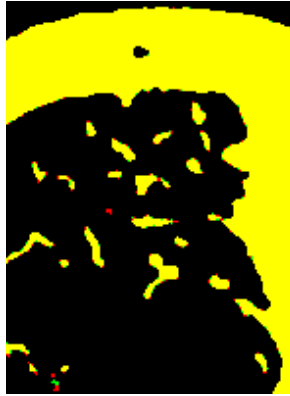

(b) DS, $a=4$, DART

rNMP $=0.003$ SSIM $=0.9859$

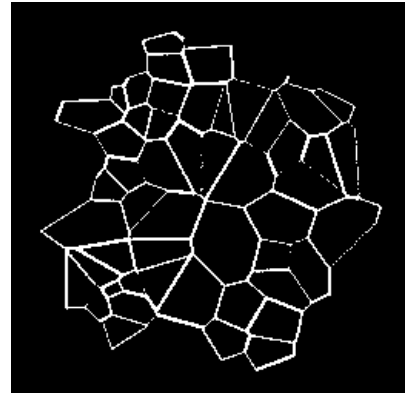

(c) $a=1$, DART

$\operatorname{avg}(\mathrm{rNMP})=0.347$ $\operatorname{avg}(\mathrm{SSIM}=0.9981)$

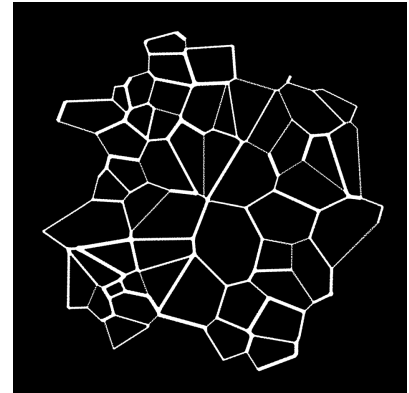

(d) DS, $a=8$, DART

$\operatorname{avg}(\mathbf{r N M P})=\mathbf{0 . 1 0 7}$ $\operatorname{avg}($ SSIM $)=0.9997$

Fig. 13. (a) and (b) Detector supersampling for phantom Fig. 10. (c) and (d) Detector supersampling for phantom Fig. 10(c).

with the DART method. Projection data was analytically simulated for two sets of phantoms: a single ring [Fig. 7(b)], with varying thickness $\Delta q \in\left[\frac{1}{16}, 4\right]$; and a foam-like structure [Fig. 7(c)], where the thickness of the cell edges was also varied from $\Delta q \in\left[\frac{1}{16}, 4\right]$. The number of projection directions was varied from 2 to 180 and data was generated for 64 detectors of width $\Delta t=1$ (hence, $R_{p}=q$ in this experiment).

DART reconstructions were performed with various levels of DS. These reconstructions were validated with the rNMP metric, measured against a high resolution rasterization of the phantom. In Fig. 9(a)-(c) and (e)-(g), the resulting rNMP values are plotted as a function of the number of projection angles for different rings. From these plots, it is clear that for low angle counts, the reconstructions greatly benefit from additional angles. However, after a certain point, "enough" information is available and additional angles offer no improved accuracy any more. This "point of sufficient information" increases as the level of upsampling is increased. The reconstruction grid then becomes finer and additional information (i.e. projection images) can then be used and smaller structures can be reconstructed with improved accuracy.

In Fig. 9(d) and Fig. 9(h), the minimal $R_{p}$ that can be reconstructed with rNMP $<0.30$ is plotted as a function of the number of projections, for increasing levels of upsampling. To be able to reconstruct the smallest rings or foam edges, a large upsampling factor is clearly required and more projection angles must be used.

Other simulated phantoms: Experiments were also performed on the simulated datasets presented in Fig. 10. The three bone phantoms [Fig. 10(a), (b), (d)] are $1024 \times 1024$ pixel phantoms based on actual reconstructions of rat femurs and where $R_{v}=\frac{1}{4}$, i.e. $\Delta s=\frac{\Delta t}{4}$. Fig. 10 represents a set of 20 randomly generated, analytically defined polyurethane foam phantom images. The width of each cell wall was chosen randomly in the interval $\left[\frac{\Delta t}{2}, \frac{5 \Delta t}{2}\right]$. Fig. 10(a), (c) are binary 
images whereas Fig. 10(b), (d) contain three distinct grey level values. It should be noted that the number of grey levels to be used in the reconstruction should not be too high. Otherwise, the prior knowledge is no longer sufficiently strong to optimally restrict the solution space. We have found that, depending on the shape of the objects, typically up to 4 or 5 unique grey levels may be present.

For each dataset, projection data was generated on a parallel beam projection geometry with 180 equiangular projection angles and 256 detector cells with $\Delta t=1$. For the pixel based phantoms [Fig. 10(a), (b), (d)], the PVE was induced by simulating high resolution projection data (with 1024 detector cells with $\Delta t=\frac{1}{4}$ ) in the intensity domain (i.e. $I$, Eq. (3)). The detector bins were then summed 4 by 4 after which the resulting data was converted to the attenuation domain (i.e. to $\boldsymbol{p}$, Eq. (4)). For the analytical phantoms [Fig. 10(c)], projection data was computed analytically, inherently modelling the PVE. For every dataset, Poisson noise was applied; the intensity of which is defined by the incident beam intensity, $I_{0}$. In these experiments, $I_{0}=20000$.

To quantify the segmentation accuracy, the rNMP measure was computed. As the experiments were performed at varying pixel or voxel sizes, the reconstructions were first rescaled to the size of the original, high resolution ground truth images. For the analytical phantoms, high resolution rasterizations were used as the ground truth. For phantoms Fig. 10(b) and (d), which contain an additional distinct grey level value representing soft-tissue, the rNMP was computed with respect to the most dense partition, i.e. the bone structures.

The following reconstruction methods were evaluated: S-SIRT [visualised for phantom Fig. 10(a) in Fig. 11(a), (c), (e)] versus DART [Fig. 11(b), (d), (f)]; no super-resolution approach ( $a=1$, Fig. 11(a), (b) versus a super-resolution approach $(a>1$, Fig. 11(c)-(f)); and sinogram upsampling [Fig. 11(c), (d)] versus detector supersampling [Fig. 11(e), (f)]. In Fig. 12, the rNMP values for these experiments are shown. The accuracy of all reconstructions has also been validated using the structural similarity (SSIM) metric [33]. These results can also be seen in Fig. 12 and provide similar insights into the performance of the methods.

For phantom Fig. 10(b) and (c), the advantage of using DART and detector supersampling can be seen in Fig. 13(a)-(d). Small trabecular structures are properly segmented only on an upsampled reconstruction grid. Similar results can be seen for the foam segmentation, where it is clear that especially the thinnest cell edges benefit the most from the proposed super-resolution approach.

Fig. 14 shows the improvement of detector supersampling on 3D DART reconstructions of phantom Fig. 10(d) for two orthogonal viewing directions. It can be seen that by applying super-resolution, the small three-dimensional trabecular structures are segmented much more accurately, also in the XZ-slices.

Limited view problem: A method to reduce the radiation dose is to reduce the number of projection angles. This, however, leads to limited data reconstruction problems.

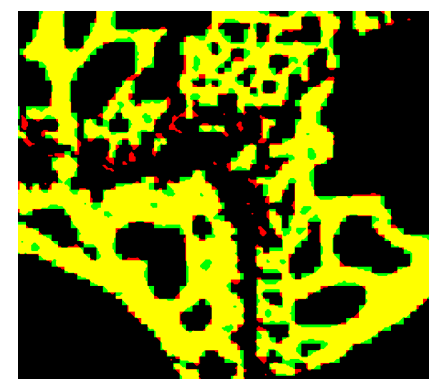

(a) $a=1$, DART, Z-axis

$\mathrm{rNMP}=0.172$

SSIM $=0.8987$

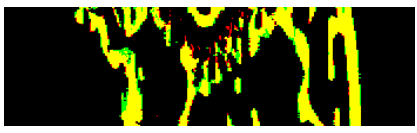

(c) $a=1$, DART, $\mathrm{X}$-axis

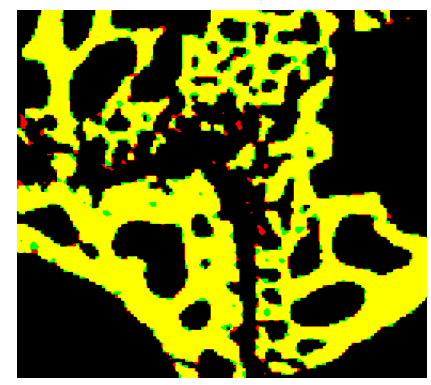

(b) $a=4$, DS, DART, Z-axis rNMP $=0.068$ SSIM $=0.9834$

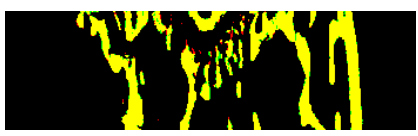

(d) $a=4$, DS, DART, X-axis
Fig. 14. The improvement of detector supersampling on 3D-DART reconstructions of phantom Fig. 10(a) is clearly visible from slices through the $\mathrm{Z}$-axis and the $\mathrm{X}$-axis.

To demonstrate the effect of this limited view problem on the proposed super-resolution, projection data of Fig. 10(b) and (c) was generated several times, with the same $I_{0}$ and the downsampling strategy as before, with a decreasing number of projection angles, effectively simulating scans with a reduced radiation dose. For each set of projection data, DART and S-SIRT reconstructions were created with and without the detector supersampling approach. The rNMP values are plotted in Fig. 15(a) and (b). One can conclude that, even with a drastically lowered number of projection angles, the combination of detector supersampling with the exploitation of prior knowledge results in reconstructed images that are more accurate than conventional S-SIRT reconstructions without a super-resolution approach and with a high number of projection angles.

Robustness of assumed principles: In the proposed superresolution approach, it is assumed that the object has a homogeneous density and that this density is known in advance. A study was performed to investigate what happens if one of these assumptions are only approximately satisfied.

To demonstrate the robustness of our algorithm with respect to deviations from the first assumption, each pixel of phantom Fig. 10 was multiplied with a normally distributed random number with mean $=1$. This was done multiple times with an increasing standard deviation. For each such image, projection data was generated with 30 projection angles, downsampled by a factor 4 - as explained before - and with $I_{0}=20000$. Reconstructions were made with S-SIRT and DART and with detector supersampling ( $a=1$ and $a=4$ ). In Fig. 16(a), the rNMP values are plotted in function of the standard deviation of the applied noise. While the rNMP of DART with the super-resolution approach indeed increases as the objects grows more and more inhomogeneous, improvements over the conventional methods are still achieved.

For phantom Fig. 10(a), projection data was created with 30 projection angles, again downsampled by a factor 4 and 


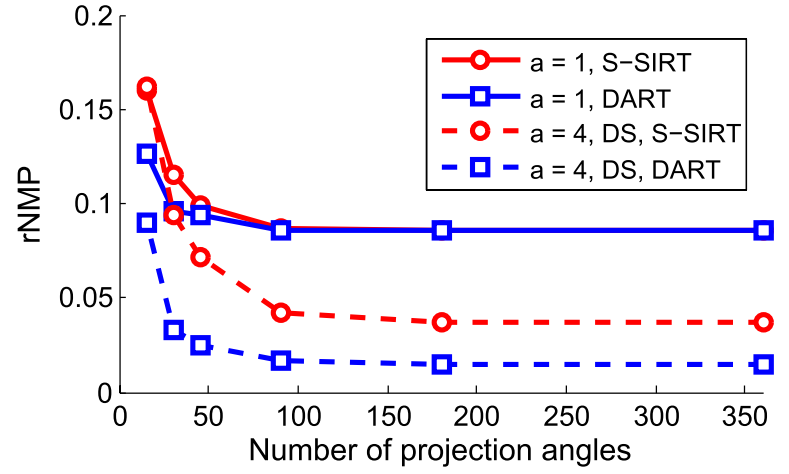

(a) phantom Fig. 10b

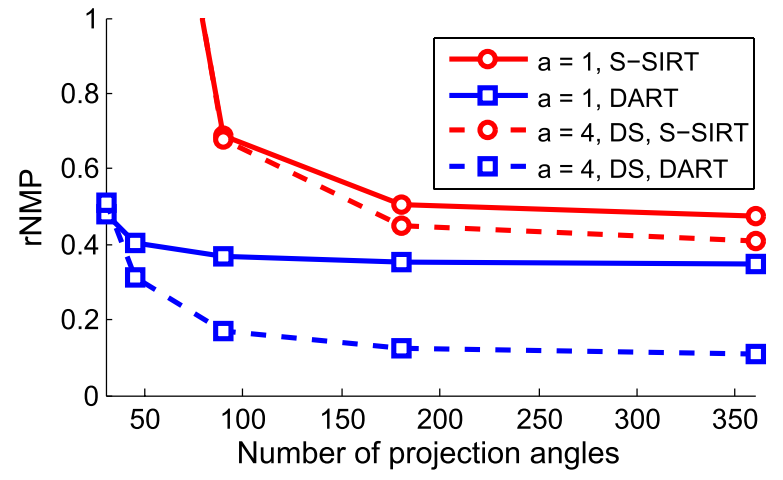

(b) phantom Fig. 10c

Fig. 15. The rNMP in function of the number of projection angles for phantoms Fig. 10(b) and Fig. 10(c) and for $a=1$ and $a=4$.

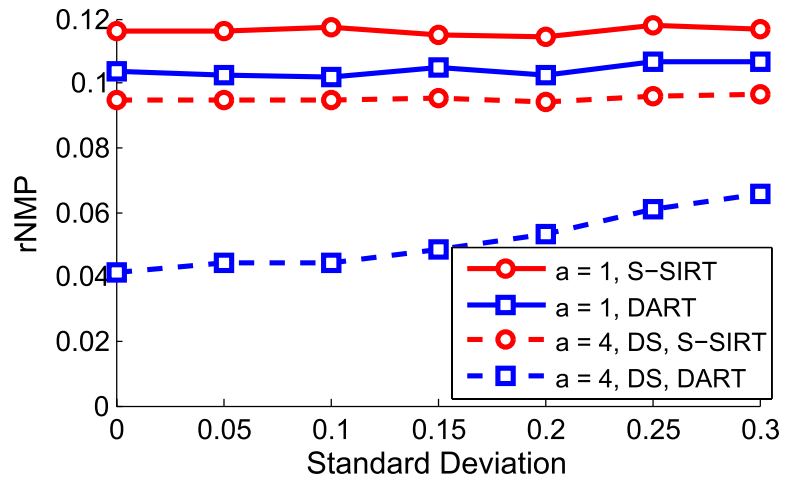

(a) phantom Fig. 10b

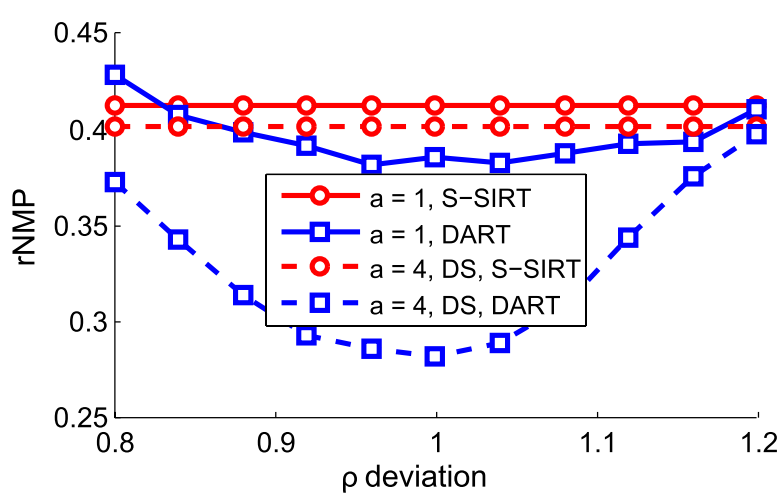

(b) phantom Fig. 10a

Fig. 16. (a) The rNMP in function of the standard deviation of the normally distributed noise that was multiplied with the phantom image Fig. 10 prior to simulating the projection data. (b) The rNMP in function of the deviation on the correct grey level $\rho$ during DART reconstructions of Fig. 10(c).

with $I_{0}=20000$. Multiple DART reconstructions were created where the assumed grey level was varied between 0.8 and 1.2 times the correct grey level. Fig. 16 plots the rNMP values for these DART reconstruction with detector supersampling $(a=1$ and $a=4)$ and for S-SIRT. It can be seen that the rNMP of the DART reconstructions indeed increases as the assumed grey level is incorrect. However, drastic improvements over the conventional S-SIRT method without superresolution can be achieved even if the chosen grey level is just an approximation of the correct grey level.

In [16] and [17], methods were proposed to automatically estimate the optimal grey levels of piece homogeneous objects.

\section{B. Real-World Experiments}

The proposed method was applied to real $\mu \mathrm{CT}$ data. Fig. 17(a) shows an FBP reconstruction of a slice through a human mandible, which was recorded using a SkyScan $1173 \mu \mathrm{CT}$ scanner with 900 equiangular projection angles in the interval $[0, \pi)$. The detector resolution was $50 \mu \mathrm{m}$. The data was corrected for ring artefacts and beam hardening with the standard SkyScan NRecon software package. Only 100 projection angles were used in the experiments and the projection data was downsampled by summing the detector bins 4 by 4 in the intensity domain (Eq. (3)), such that many of the smaller structures were relatively small compared to the new detector sizes. Three distinct grey level values were used.
One for air, one for soft tissue and one for bone. Validation was performed using the pixels of the latter category only. Given the polychromatic nature of the X-ray spectrum, in practice, one can not know the exact grey levels of the materials in advance. In this experiment, the discrete grey levels $\rho$ were manually approximated, guided by the grey levels present in the initial SIRT reconstruction, $v^{(0)}$. If the initial reconstruction is too erroneous to accurate estimate the grey level values, e.g. when there are only a few projections, one could use one of the grey level estimation algorithms that have been presented in the literature, such as the semi-automatic DGLS method [16] or an automatic method that combines grey level estimation into DART [17].

From the FBP reconstruction [Fig. 17(a)], created with the non-downsampled and the full set of projection data, a segmentation was manually created [Fig. 17(b)]. This segmentation was used to validate the results by measuring the rNMP. This FBP reconstruction was also segmented using Otsu's clustering method [Fig. 17(c)]. Fig. 17(d)-(g) indicate that also for real datasets, the addition of super-resolution and DART significantly improves the accuracy of the segmentation. When comparing Fig. 17(c) with Fig. 17(g), it can be seen that with the proposed super-resolution approach, segmentations of low resolution projection data can be obtained that are of comparable quality to that of high resolution, high dose scans.

In Fig. 18, various reconstructions are shown of the polyurethane foam also shown in Fig. 1. In total, 


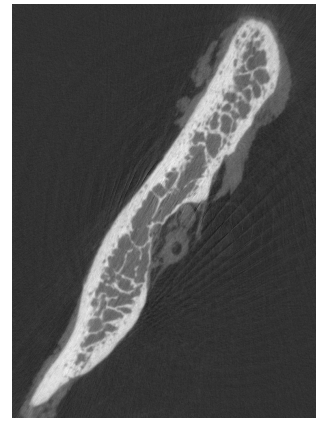

(a) $R_{p}=1, a=1$

FBP reconstruction

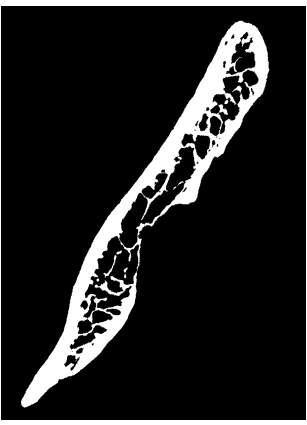

(b) $R_{p}=1, a=1$

manual segmentation

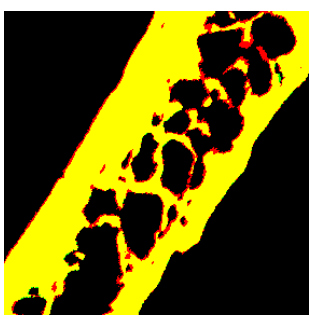

(c) $R_{p}=1$

$a=1$, FBP

rNMP=0.106

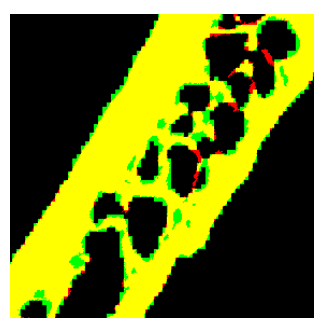

(d) $R_{p}=4$ $a=1$, S-SIRT $\mathrm{rNMP}=0.175$

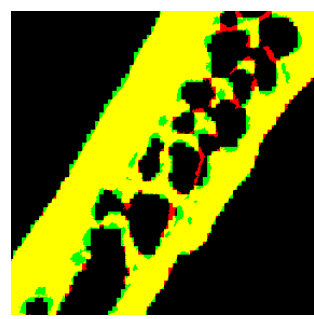

(e) $R_{p}=4$ $a=1$, DART $\mathrm{rNMP}=0.184$

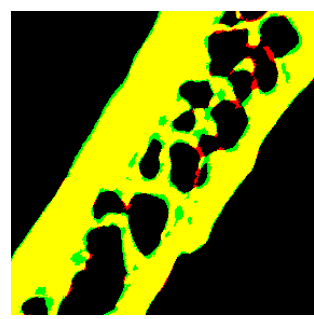

(f) $R_{p}=4$ $a=1$, DS, S-SIRT $\mathrm{rNMP}=0.171$

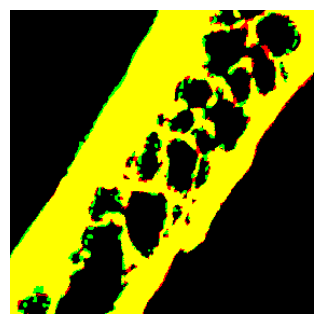

(g) $R_{p}=4$ $a=4$, DS, DART rNMP $=0.115$

Fig. 17. Results for a real $\mu \mathrm{CT}$ dataset of a slice through a human mandible.

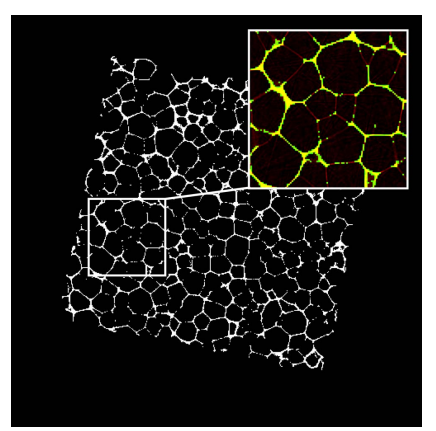

(a)

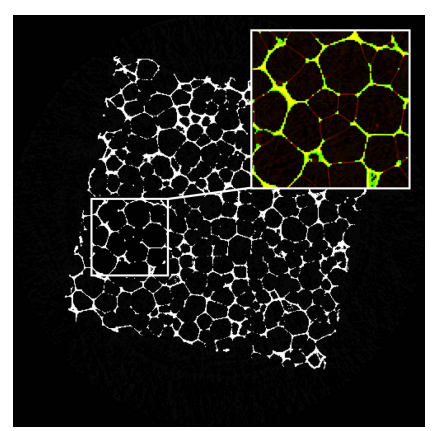

(b)

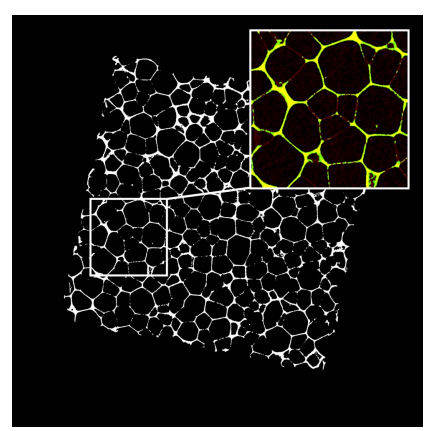

(c)

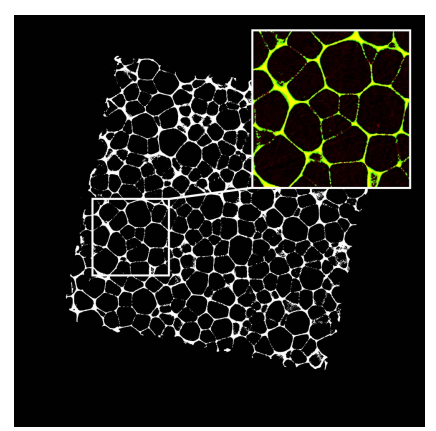

(d)

Fig. 18. Results for a real $\mu \mathrm{CT}$ dataset containing a polyurethane foam. (a) $\mathrm{a}=1$, S-SIRT. (b) $\mathrm{a}=1$, DART. (c) $\mathrm{a}=1$, DS, S-SIRT. (d) $\mathrm{a}=4$, DS, DART

500 projection images were taken in a SkyScan $1172 \mu \mathrm{CT}$ scanner at a pixel resolution of $17 \mu \mathrm{m}$. The projection data was downsampled by summing the detector bins 2 by 2 in the intensity domain and the SkyScan NRecon software package was used for ring artefact and beam hardening correction. Reconstructions were created with both S-SIRT and DART, and with $a=1$ and $a=4$ (which applies the DS superresolution technique). In cut-outs, parts of the reconstructed images (green) are overlaid with a high resolution FBP reconstruction of the foam (red). As in the previous experiments, the application of DART without the use of a super-resolution technique, does not result in improved image quality. However, when discrete tomography and super-resolution are combined, even the thin cell walls become clearly visible.

\section{CONCLUSiON}

Accurate segmentation of structures that are small with respect to the reconstruction pixel size, poses a very complex and difficult problem as reconstructed images often lack contrast due to a partial volume effect. This often means that even the intrinsic detector resolution can not be achieved. High resolution reconstructions can provide a solution, but are often not feasible due to X-ray dose limitations, limited scanning time or hardware constraints.

To improve the detection of small structures in low resolution CT acquisitions, the use of a super-resolution approach has been proposed in which reconstructed images are computed on an upsampled reconstruction grid. Two geometrical methods for achieving super-resolution have been investigate. In sinogram upsampling, the projection data is upsampled by linear interpolation, which (often wrongly) assumes that the projection images are smooth. In detector supersampling, multiple rays per detector element are cast through the reconstruction grid and no interpolation is necessary. Both methods result in a limited data problem. It was shown that with discrete tomography (DART) prior knowledge about the object 
materials, can be exploited to overcome this problem and thus increase the resolution of the reconstruction. Previously, DART has only been used to reduce the number of projection angles.

Experiments were performed on simulated as well as real data of objects containing small structures. Without using a super-resolution technique on objects containing small structures, the addition of prior knowledge (DART) sometimes resulted in less accurate segmentations when compared to the conventional S-SIRT algorithm. This effect was predicted in Fig. 5, where it was noted that DART is only suited for objects that are large with respect to the pixel size. However, if a super-resolution technique was applied, the use of prior knowledge with the DART method clearly resulted in more accurate reconstructions than the conventional S-SIRT approach. This effect was observed to be generally more profound if detector supersampling was chosen over sinogram upsampling.

In conclusion, the use of the detector supersampling superresolution technique in which prior knowledge about the object density is exploited, can effectively increase the spatial resolution of a reconstructed image. In that way, small structures can be segmented more accurately with a shorter scan time and a lower radiation dose.

\section{REFERENCES}

[1] N. Otsu, "A threshold selection method from gray level histograms," IEEE Trans. Syst., Man, Cybern., vol. 9, no. 1, pp. 62-66, Mar. 1979.

[2] P. L. Salmon and A. Y. Sasov, Advanced Bioimaging Technologies in Assessment of the Quality of Bone and Scaffold Materials. New York, NY, USA: Springer-Verlag, 2007

[3] A. Parfitt, M. Drezner, F. Glorieux, J. Kanis, H. Malluche, P. Meunier, et al., "Bone Histomorphometry: Standardization of nomenclature, symbols and units," J. Bone Mineral Res., vol. 2, no. 6, pp. 595-610, 1987.

[4] E. Perilli, V. Le, B. Ma, P. Salmon, K. Reynolds, and N. L. Fazzalari, "Detecting early bone changes using in vivo micro-ct in ovariectomized, zoledronic acid-treated, and sham-operated rats," Osteoporosis Int., vol. 21, no. 8, pp. 1371-1382, 2009.

[5] S. Bals, K. J. Batenburg, J. Verbeeck, J. Sijbers, and G. Van Tendeloo, "Quantitative Three-Dimensional reconstruction of catalyst particles for bamboo-like carbon nanotubes," Nano Lett., vol. 7, no. 12, pp. 3669-3674, 2007.

[6] S. C. Park, M. K. Park, and M. G. Kang, "Super-resolution image reconstruction: A technical overview," IEEE Signal Process. Mag., vol. 20, no. 3, pp. 21-36, May 2003.

[7] J. A. Kennedy, O. Israel, A. Frenkel, R. Bar-Shalom, and H. Azhari, "Super-resolution in PET imaging," IEEE Trans. Med. Imag., vol. 25, no. 2, pp. 137-147, Feb. 2006.

[8] G. Marai, D. Laidlaw, and J. Crisco, "Super-resolution registration using tissue-classified distance fields," IEEE Trans. Med. Imag., vol. 25, no. 2, pp. 177-187, Feb. 2006.

[9] A. Patil, R. Singhai, and J. Singhai, "Curvelet transform based superresolution using sub-pixel image registration," in Proc. 2nd CEEC, Sep. 2010, pp. 1-6.

[10] E. Sidky, Y. Duchin, X. Pan, and C. Ullberg, "A constrained, totalvariation minimization algorithm for low-intensity X-ray CT," Med. Phys., vol. 38, p. S117, Jul. 2011.

[11] M. Li, H. Yang, and H. Kudo, "An accurate iterative reconstruction algorithm for sparse objects: Application to 3D blood vessel reconstruction from a limited number of projections," Phys. Med. Biol., vol. 47, no. 15, pp. 2599-2609, 2010.

[12] E. Sidky and X. Pan, "Image reconstruction by constrained, totalvariation minimization," Phys. Med. Biol., vol. 53, no. 2, pp. 4777-4807, 2008.

[13] J. Bian, J. H. Siewerdsen, X. Han, E. Y. Sidky, J. L. Prince, C. A. Pelizzari, et al., "Evaluation of sparse-view reconstruction from flat-panel-detector cone-beam CT," Phys. Med. Biol., vol. 55, no. 22, pp. 6575-6599, 2010.

[14] K. J. Batenburg and J. Sijbers, "DART: A practical reconstruction algorithm for discrete tomography," IEEE Trans. Image Process., vol. 20, no. 9, pp. 2542-2553, Sep. 2011.
[15] J. Gregor and T. Benson, "Computational analysis and improvement of SIRT," IEEE Trans. Med. Imag., vol. 27, no. 7, pp. 918-924, Jul. 2008.

[16] K. J. Batenburg, W. van Aarle, and J. Sijbers, "A semi-automatic algorithm for grey level estimation in tomography," Pattern Recognit. Lett., vol. 32, no. 9, pp. 1395-1405, Jul. 2011.

[17] W. van Aarle, K. Batenburg, and J. Sijbers, "Automatic parameter estimation for the discrete algebraic reconstruction technique (DART)," IEEE Trans. Image Process., vol. 21, no. 11, pp. 4608-4621, Nov. 2012

[18] S. Van Aert, K. J. Batenburg, M. D. Rossell, R. Erni, and G. Van Tendeloo, "Three-dimensional atomic imaging of crystalline nanoparticles," Nature, vol. 470, no. 7334, pp. 374-377, 2011.

[19] E. Van de Casteele, K. J. Batenburg, P. Salmon, and J. Sijbers, "Bone segmentation using discrete tomography," in Proc. Micro-CT User Meeting, 2011, pp. 1-12.

[20] T. Roelandts, K. J. Batenburg, E. Biermans, C. Kubel, S. Bals, and J. Sijbers, "Accurate segmentation of dense nanoparticles by partially discrete electron tomography," Ultramicroscopy, vol. 114, pp. 96-105, Mar. 2012.

[21] W. van Aarle, G. Van Gompel, K. J. Batenburg, E. Van de Casteele, and J. Sijbers, "A 3-dimensional discrete tomography approach for superresolution micro-CT images: Application to foams," in Proc. 1st Int. Conf. Image Formation X-ray Comput. Tomogr., 2010, pp. 45-48.

[22] G. Van Gompel, K. J. Batenburg, E. Van de Casteele, W. van Aarle, and J. Sijbers, "A discrete tomography approach for superresolution microCT images: Application to bone," in Proc. IEEE Int. Symp. Biomed. Imag., From Nano Macro, Apr. 2010, pp. 816-819.

[23] K. J. Batenburg, W. Fortes, L. Hajdu, and R. Tijdeman, "Bounds on the quality of reconstructed images in binary tomography," Discrete Appl. Math., vol. 161, no. 15, pp. 2236-2251, 2013.

[24] P. M. Joseph and C. Ruth, "A method for simultaneous correction of spectrum hardening artifacts in CT images containing both bone and iodine," Med. Phys., vol. 24, pp. 1629-1634, Oct. 1997.

[25] E. Van de Casteele, D. Van Dyck, J. Sijbers, and E. Raman, "A modelbased correction method for beam hardening Artefacts in X-ray microtomography," J. X-ray Sci. Technol., vol. 12, no. 1, pp. 53-57, 2004.

[26] M. Krumm, S. Kasperl, and M. Franz, "Reducing non-linear artifacts of multi-material objects in industrial 3D computed tomography," Nondestruct. Test. Eval., vol. 41, no. 4, pp. 242-251, 2008.

[27] G. Van Gompel, K. Van Slambrouck, M. Defrise, K. J. Batenburg, J. de Mey, J. Sijbers, et al., "Iterative correction of beam hardening artifacts in CT," Med. Phys., vol. 38, pp. S361-S3614, Jul. 2011.

[28] A. C. Kak and M. Slaney, Principles of Computerized Tomographic Imaging, R. Cotellessa, Ed. Piscataway, NJ, USA: IEEE Press, 1988.

[29] G. T. Herman and A. Kuba, Discrete Tomography: Foundations, Algorithms, and Applications. Cambridge, MA, USA: Birkhäuser, 1999.

[30] G. T. Herman and A. Kuba, Advances in Discrete Tomography and its Applications. Cambridge, MA, USA: Birkhäuser, 2007.

[31] K. J. Batenburg, S. Bals, J. Sijbers, C. Kübel, P. A. Midgley, J. C. Hernandez, et al., "3D imaging of nanomaterials by discrete tomography," Ultramicroscopy, vol. 109, no. 6, pp. 730-740, 2009.

[32] K. J. Batenburg, J. Sijbers, H. F. Poulsen, and E. Knudsen, "DART: A robust algorithm for fast reconstruction of three-dimensional grain maps," J. Appl. Crystalogr, vol. 43, no. 6, pp. 1464-1473, 2010.

[33] Z. Wang, A. C. Bovik, H. R. Sheikh, and E. P. Simoncelli, "Image quality assessment: From error visibility to structural similarity," IEEE Trans. Image Process., vol. 13, no. 4, pp. 600-612, Apr. 2004

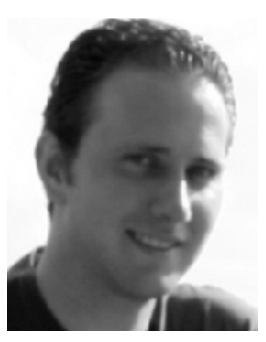

Wim van Aarle received the Degree in computer science from the University of Antwerp, Belgium, in 2007. He became a Researcher with the Vision Laboratory, University of Antwerp. $\mathrm{He}$ received the Ph.D. degree in science in 2012. $\mathrm{He}$ was a Post-Doctoral Fellow with the Intel ExaScience Laboratory, Leuven, Belgium, and the European Synchrotron Radiation Facility, Grenoble, France. His research interests are focused on high performance computing and on discrete tomography in a wide spectrum of applications. 


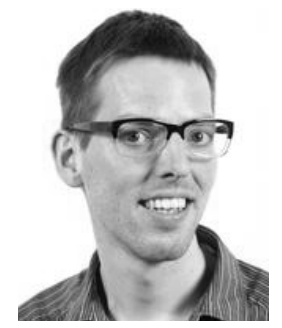

Kees Joost Batenburg received the M.Sc. degree in mathematics and the M.Sc. degree in computer science from the University of Leiden, Leiden, The Netherlands, in 2002 and 2003, respectively, and the $\mathrm{Ph} . \mathrm{D}$. degree in mathematics in 2006. He is currently a Senior Researcher with Centrum Wiskunde en Informatica, Amsterdam, where he performs research in the field of computational imaging. He has two part-time professorships at the University of Antwerp, Belgium, and the University of Leiden, The Netherlands.

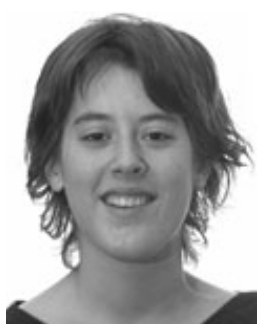

Gert Van Gompel received the Degree in physics from the University of Antwerp and the Ph.D. degree from the Vision Laboratory, University of Antwerp, in 2009. Her work entitled "Towards accurate image reconstruction from truncated X-ray CT projections." In 2010, she was a Medical Physicist with the University Hospital of Brussels, Belgium. In 2013, she received the Postgraduate degree in medical radiation physics from Katholieke Universiteit Leuven, Belgium.

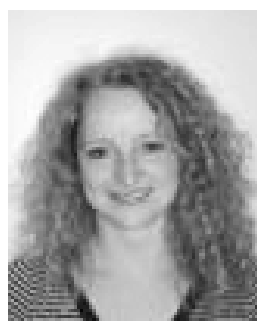

Elke Van de Casteele received the Degree in physics from the University of Antwerp in 1998, and the Ph.D. degree from the Vision Laboratory, University of Antwerp, in 2004. Her work entitled "Model-based approach for beam hardening correction and resolution measurements in microtomography." She was with SkyScan, an SME specialized in the development and manufacturing of X-ray microand nano-CT systems. In 2010, she was a PostDoctoral Researcher with the University of Antwerp.

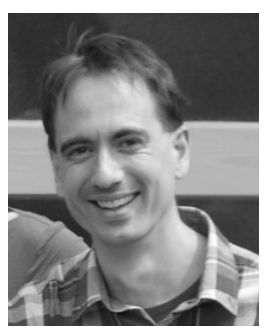

Jan Sijbers received the B.Sc. degree in physics from the University of Hasselt, Diepenbeek, Belgium, in 1991, and the M.S. degree in physics and the Ph.D. degree in sciences/physics from the University of Antwerp, Antwerp, Belgium, in 1993 and 1998, respectively. Currently, he is a Full Professor with the Physics Department, University of Antwerp. His research is focused on biomedical image reconstruction, processing, and analysis. 